\title{
Conduta na lesão intraepitelial de alto grau em mulheres adultas
}

\section{Management of high grade squamous intraepithelial lesion of the cervix of adult women}

\author{
Pauliana Sousa Oliveira'; Camila Cardoso Coelho'; Elza Francinet Siqueira de Cerqueira'; Flávia Verônica Ferreira lopes'; Minell
}

Salles Alves Fernandes ${ }^{1}$; Denise leite Maia Monteiro²

\section{R E S U M O}

\begin{abstract}
O câncer cervical é a segunda causa mais comum de câncer entre as mulheres no mundo, apesar de apresentar grande potencial de prevenção e cura quando diagnosticado e tratado precocemente, o que pode reduzir a taxa de mortalidade entre as acometidas. Como não há consenso entre as medidas terapêuticas nas lesões intraepiteliais escamosas cervicais de alto grau (HSIL), buscamos discutir a conduta frente às mulheres adultas que apresentam HSIL e o seguimento após o procedimento adotado. Foi realizada pesquisa eletrônica no Medline (através do PubMed), National Guidelines Clearinghouse, Google Acadêmico e Lilacs. Os consensos identificados foram avaliados segundo sua validade e recomendações. Em relação ao acompanhamento após o tratamento, 0 Europeu acompanha por citologia de 6/6 meses; o Australiano, citologia e colposcopia de 6/6 meses; o Americano preconiza a realização da captura híbrida em seis a 12 meses ou citologia em seis meses. Já o Projeto Diretrizes do Brasil recomenda que reavaliações clínicas e colpocitológicas devam ser realizadas a cada três ou quatro meses nos primeiros dois anos de seguimento. Estudos comparando o método "Ver e Tratar" com os três passos (histologia, colposcopia, biópsia) concluíram que este último é indicado em mulheres LSIL/ASCUS antes de se submeterem à exérese da zona de transformação (EZT), enquanto que o "Ver e Tratar" é indicado em mulheres com HSIL comprovada na citologia e sugestiva na colposcopia, pois apresenta como vantagens, o baixo custo e a resolução imediata. Todos os consensos são unânimes ao afirmar que frente à HSIL comprovada, retira-se a lesão por meio de ablação ou conização ou EZT.
\end{abstract}

Descritores: Neoplasia intra-epitelial cervical. Detecção precoce de câncer. Terapêutica. Conização. Mulheres.

\section{INTRODUÇÃO}

$\mathrm{O}$ câncer cervical é a segunda causa mais comum de câncer entre as mulheres no mundo, apesar de apresentar grande potencial de prevenção e cura quando diagnosticado e tratado precocemente, o que pode reduzir a taxa de mortalidade entre as acometidas ${ }^{1}$. Estima-se que $75 \%$ das pessoas sexualmente ativas irão se infectar pelo Papilomavírus humano (HPV), o agente causal do câncer de colo uterino, em algum ponto de suas vidas².

Estudos demonstraram que surgem 500 mil casos novos de câncer do colo uterino por ano no mundo, sendo este, o responsável pelo óbito de aproximadamente 230.000 mulheres por ano. Sua incidência torna-se evidente na faixa etária de 20 aos 29 anos e o risco aumenta até atingir seu pico geralmente na faixa etária de 45 aos 49 anos $^{3}$. No Brasil, as estimativas do Instituto Nacional do Câncer (INCA) para o ano de 2010 e 2011, apontam que devam ocorrer 18.430 casos novos de câncer do colo do útero, com risco estimado de 18 casos a cada 100.000 mulheres ${ }^{1}$.
A forma mais simples de rastreá-lo é através da realização do teste de Papanicolaou que, de acordo com as diretrizes brasileiras, deve ser realizado em mulheres de 25 a 60 anos de idade ou que já tenham atividade sexual mesmo antes desta faixa de idade 4 .

No entanto, diante de sugestiva lesão intraepitelial de alto grau (HSIL), procede-se à investigação a partir dos três passos: citologia, colposcopia e biópsia. Para o estudo histopatológico, a fim de evitar a progressão para lesões mais graves ou para carcinoma de colo do útero, pode ser feita a cirurgia de alta freqüência ${ }^{5}(\mathrm{CAF})$, atualmente denominada exérese da zona de transformação (EZT), também conhecida como LLETZ (Large Loop Excison of the Transformation Zone) ou LEEP (Loop Electrosurgical Excision Procedure), que consiste em procedimento de baixo custo, podendo ser realizado no ambulatório ou consultório, substituindo outro meio de remoção, as conizações, dependendo das características e localização das lesões.

O objetivo deste estudo é discutir a melhor conduta frente às mulheres adultas que apresentam HSIL, visto que não há consenso entre as medidas terapêuticas di-

Trabalho realizado no Departamento de Ginecologia do UNIFESO - RJ-BR.

1. Acadêmicas do Curso de Graduação de Medicina da UNIFESO - Teresópolis - RJ-BR; 2. Professora Titular do Centro Universitário Serra dos Órgãos (UNIFESO)-Teresópolis-RJ-BR. 
ante de cada uma delas, tomando como relevância os riscos e benefícios de cada procedimento e seu potencial de cura.

\section{MÉTODOS}

Foi realizada pesquisa eletrônica de publicações utilizando o Medline (através do PubMed), National Guidelines Clearinghouse, Google Acadêmico e LILACS. Os consensos identificados e, especificamente relacionados ao tema, foram avaliados segundo sua validade e suas recomendações, criticados e sumarizados. Para identificação de publicações no PubMed, foi utilizada a seguinte estratégia de busca ("squamous intraepithelial lesion" OR "SIL") OR ("cervical intraepithelial neoplasia/ therapy"[Mesh] AND (" LEEP" OR "LLETZ"). Ainda utilizou-se informações relevantes de publicações brasileiras. A seguir, realizou-se a análise de todas as recomendações obtidas.

\section{RESULTADOS}

Em mulheres adultas com HSIL, em que não é possivel identificar NIC 2 ou 3 histologicamente, tanto o diagnóstico excisional quanto a observação por colposcopia e citologia, em intervalos de seis meses, durante um ano, é aceitável. Depois de um ano de observação, mulheres com dois resultados negativos consecutivos para SIL ou malignidade podem retornar para o rastreio citológico de rotina. O diagnóstico excisional é recomendado para muIheres com HSIL em que a colposcopia é insatisfatória, exceto em populações especiais como mulheres grávidas ${ }^{6}$.

A abordagem terapêutica dessas pacientes é assunto bastante discutido. Quando não são tratadas, as neoplasias intraepiteliais cervicais podem progredir para lesões mais graves ou carcinoma invasor. Essa progressão dependerá em parte do grau ou gravidade da lesão. Quanto maior o grau de anormalidade do resultado do exame histológico, maior será o índice de progressão para um tipo de lesão mais severa e mais rápida será a evolução deste processo. Por essa razão, enquanto o NIC 1 pode ser conduzido apenas por observação, o NIC 3 tem que ser tratado pela completa destruição do epitélio anormal, preferencialmente excisão da cérvice ou conização ${ }^{7}$. Muitas mulheres com NIC 2 apresentarão regressão das suas lesões sem tratamento. Na verdade, o significado do NIC 2 não é claro, o risco de progressão para NIC 3 e câncer parece ser maior em mulheres com NIC 2 do que para mulheres com NIC $1^{8}$.

A excisão da cérvice pode ser realizada de várias formas, porém os métodos comumente utilizados são a conização e o (LEEP). Ambos os métodos proporcionam material para avaliação histopatológica. Entretanto o LEEP se tornou mais popular, pois pode ser realizado com míni- ma perda sanguínea9 . O Consenso Europeu, utiliza LLETZ e LEEP como sinônimos ${ }^{10}$, o que também adotaremos. No Brasil, foi denominada CAF e atualmente utiliza-se o termo EZT.

A escolha do tratamento para mulheres com HSIL dependerá do diagnóstico suspeito, do tamanho e do tipo da zona de transformação, do risco de evolução da lesão, da idade e da preservação da fertilidade ${ }^{10}$. Porém, há estudos que afirmam haver diferenças significativas de acordo com a idade, paridade ou o tipo de parto entre as pacientes que se submeteram ao LLETZ ou à conização ${ }^{11,12}$.

\section{DISCUSSÃO}

As indicações para conização e LLETZ incluem: NIC comprovado por biópsia; discrepância entre os achados citológicos, colposcópicos e de biópsia ou suspeita de lesões microinvasivas ${ }^{12}$. De acordo com o Consenso Australiano de 2005, incluem-se também: falha na visualização da zona de transformação cervical ou colposcopia insatisfatória, suspeita de câncer invasivo inicial na citologia, colposcopia ou biópsia e suspeita da presença adicional de anormalidade glandular significativa na citologia ou biópsia, isto é, lesão mista.

Ainda não se adotou padrão único para abordagem terapêutica de mulheres com HSIL. Segundo o Consenso Europeu de 2006 (Figura 1), se a interpretação citológica de HSIL é confirmada, a excisão da zona de transformação (LLETZ) é recomendada, desde que a mulher não esteja grávida ${ }^{13}$. Se a colposcopia é insatisfatória, a presença de uma lesão localizada na endocérvice tem que ser excluída, além disso, o diagnóstico excisional da zona de transformação ou conização deve ser realizado.

O Consenso da American Society for Colposcopy and Cervical Pathology (ASCCP) determina que em muIheres com NIC de alto grau, o tratamento cirúgico é recomendado e a conduta expectante não é indicada (Figura 2). A ablação ou destruição local, usando laser, crioterapia, coagulação a frio ou diatermia radical são estratégias de conduta adequadas caso a colposcopia seja satisfatória. No caso de recorrência ou quando a colposcopia é insatisfatória, a excisão por meio de LLETZ ou conização deve ser escolhida ${ }^{13,14}$.

A American Congress of Obstetricians and Gynecologists (ACOG) define que, em mulheres acima de 21 anos com resultados citológicos de HSIL, o LLETZ imediato ou a colposcopia com avaliação endocervical são abordagens aceitáveis (Figura 2). Um procedimento diagnóstico excisional deve ser realizado em todas as mulheres com HSIL, desde que não estejam grávidas, quando a colposcopia for insatisfatória ou quando NIC, de qualquer nível, for identificado na avaliação endocervical ${ }^{1}$.

De acordo com o Consenso Australiano (Figura 1), o tratamento ablativo é recomendado, caso HSIL seja confirmada histologicamente, e pode ser realizado através 


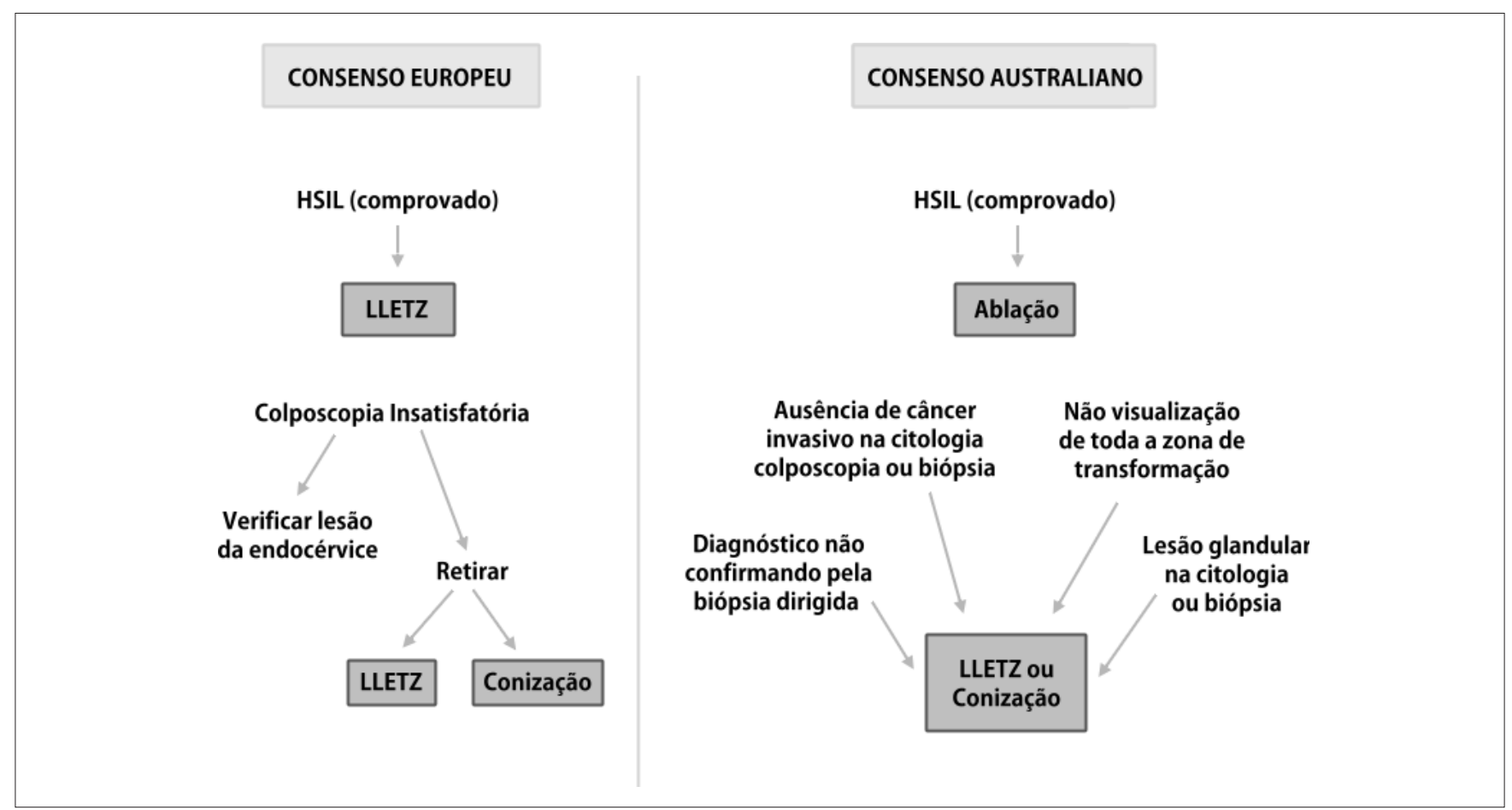

Figura 1 - Recomendações do Consenso Europeu (2006) e Australiano (2005).

\section{CONSENSO AMERICANO}

\section{ASCCP}

NIC 2 ou 3

Tratamento Cirúrgico

\section{Colposcopia Satisfatória}

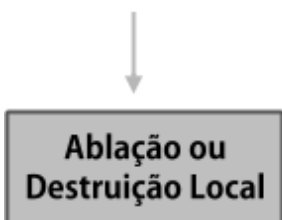

\section{Colposcopia} Insatisfatória

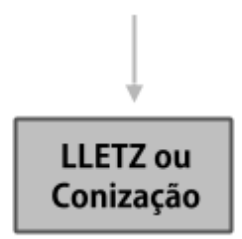

\section{ACOG}

HSIL
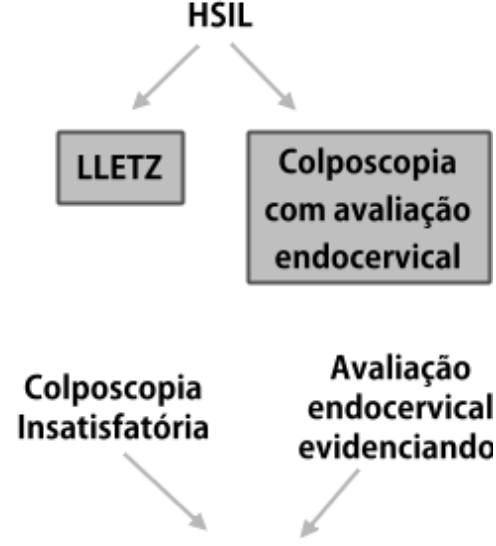

\section{Diagnóstico} excisional

Figura 2 - Consenso da ASCCP 2006/7 e ACOG 2008.

de laser de dióxido de carbono, alem das técnicas anteriormente citadas. Porém esse tratamento requer como condições: biópsia dirigida que confirme o diagnóstico, nenhuma evidência de câncer invasivo na citologia, colposcopia ou biópsia, visualização de toda a zona de transformação cervical e ausência de lesão glandular na citologia ou biópsia. Caso tais condições não se apliquem, o tratamento excisional deve ser realizado (LLETZ ou conização). 
Quanto à abordagem terapêutica de mulheres acima de 45 anos, tanto o Consenso Australiano quanto Shin et al. ${ }^{12}$, concordaram que a melhor opção é a conização, pois este parece alcançar mais margens cirúrgicas negativas para a lesão do que o LLETZ.

Em termos de eficácia, não há tratamento cirúrgico conservador superior para o tratamento da NIC. Todas as mulheres tratadas para NIC 1, 2 ou 3, requerem acompanhamento regular. O tratamento com procedimentos excisionais tem a vantagem óbvia de permitir a avaliação histológica da biópsia. O exame histológico de toda a zona de transformação permite a avaliação do estado das margens e a exclusão de doença microinvasiva ou glandular. Mulheres com maior risco de doença residual ou recorrente devem ser consideradas para abordagem mais intensa após o tratamento. Estas são as pacientes com lesão de alto grau, idade superior a 40 anos, com lesão glandular ou alterações ao exame histológico das margens excisionadas ${ }^{10}$. As diretrizes européias preconizam que mulheres tratadas para HSIL, requerem acompanhamento citológico aos seis, 12 e 24 meses e, posteriormente, citologia anual por mais cinco anos, até voltar ao rastreio no intervalo de rotina. A colposcopia deve ser realizada juntamente com a citologia na visita de acompanhamento do sexto mês ${ }^{15}$.

De acordo com o Projeto Diretrizes do Brasil ${ }^{16}$, o acompanhamento adequado das pacientes é importante, principalmente nos dois primeiros anos imediatos à terapêutica, visto que, $50 \%$ das recorrências, são detectadas no primeiro ano de seguimento e $85 \%$ com dois anos. Em cinco anos de seguimento, 95\% das recidivas terão sido detectadas. Alguns grupos de pacientes podem se beneficiar com seguimento sistemático após o tratamento ${ }^{17}$. Tal Consenso recomenda ainda que as reavaliações clínicas e colpocitológicas devam ser realizadas a cada três ou quatro meses, nos primeiros dois anos de seguimento, ampliando para intervalos de seis meses do terceiro ao quinto ano de acompanhamento. A partir de então o seguimento pode ser realizado anualmente ${ }^{18}$.

O consenso australiano aponta como pacientes de maior risco para desenvolver furturamente lesão de alto grau ou câncer cervical, as mulheres acima de 50 anos ao tratamento incial| ${ }^{19,20}$, mulheres que demonstram persistência da infecção cervical por tipos de HPV de alto-risco ${ }^{21-24}$, aquelas que foram tratadas por técnicas excisionais e naquelas em que as margens excisionadas foram consideradas comprometidas ${ }^{19}$.

Este mesmo consenso preconiza que mulheres tratadas por HSIL previamente requerem colposcopia e citologia cervical quatro a seis meses após o tratamento. A citologia cervical e a tipagem do HPV devem ser, então, realizadas 12 meses após o tratamento e, depois disso, anualmente, até que resultados dos testes sejam negativos em duas ocasiões consecutivas. Em seguida a mulher deve ser acompanhada como a população comum $21-23,25-28$.
Segundo o Consenso da ACOG de 2008 (Figura 1), a conduta após o tratamento da HSIL para mulheres adultas inclui o teste do HPV-DNA, em seis a 12 meses, ou apenas citologia em seis meses ou a combinação de citologia e colposcopia no intervalo de seis meses.

De acordo com os dois estudos levantados quanto à melhor estratégia de detecção, tratamento da lesão de alto grau e seguimento após procedimento invasivo, para evitar recorrência ou desenvolvimento de carcinoma invasor do colo uterino, HanByoul Cho e Jae-Hoon Kim²9, compararam o método "Ver e tratar" com o método dos três passos (citologia, colposcopia e biópsia), após revisão de 1011 prontuários de pacientes submetidas à CAF no Departamento de Obstetrícia e Ginecologia do Hospital Gangnam Severance, de janeiro de 2004 a dezembro de 2008 , sendo que foram submetidas à colposcopia aquelas com citologia anormal ou que, mesmo sem alteração citológica, apresentavam teste HPV positivo e persistente (definidos por eles como dois ou mais testes positivos, com intervalo de seis meses entre as amostras). Concluíram que o método "Ver e tratar" é estratégia eficiente em mulheres com HSIL comprovada na citologia e que tenham tal resultado sugerido na colposcopia, como também por ser procedimento de baixo custo, além de propiciar à mulher resolução mais imediata. Porém, ponderam que deva ser usado apenas por colposcopistas experientes a fim de evitar o tratamento excessivo. Já em relação aos três passos, indicaram naquelas com LSIL/ASCUS antes de se submeterem à cirurgia de alta frequência.

Já o estudo de Park et al. ${ }^{30}$, analisou o teste de HPV aplicado para acompanhamento de pacientes submetidas à conização diante de HSIL. Eles buscaram determinar se o teste de HPV após conização é fator preditivo para recorrência/ persistência das lesões após conização a frio ou LLETZ. Tal teste foi realizado entre três e seis meses pós-conização (diferente do que propõe a ACOG, a qual sugere que o teste seja feito, pelo menos, seis meses depois do procedimento), sendo que o intervalo de tempo médio entre a conização e a recorrência foi cinco meses. Concluíram então que, a aplicação do teste de HPV entre três e seis meses é importante para predizer a persistência ou recorrência das lesões, além da investigação das pacientes com teste positivo ser ainda mais meticulosa.

\section{CONSIDERAÇÕES FINAIS}

Todos os consensos são unânimes ao afirmar que frente à presença de lesão intraepitelial de alto grau em mulheres adultas, a excisão desta deve ser feita.

O tratamento excisional é recomendado caso NIC 2/3 ou HSIL persista por dois anos após o diagnóstico inicial, ou se NIC 2/3 for diagnosticado histologicamente deve ser feito por LLETZ. Caso seja detectado NIC 3 ou carcinoma in situ a qualquer momento, o tratamento também é indicado. 
Em mulheres adultas com HSIL em que não é possivel identificar NIC 2 ou 3 histologicamente, tanto o diagnóstico excisional quanto a observação por colposcopia e citologia em intervalos de seis meses durante um ano, é aceitável.

A excisão da cérvice pode ser realizada de várias formas, porém os métodos comumente utilizados são a conização e o LLETZ.
Enfim, todas as formas de tratamento explanadas e as diferenças entre os seguimentos pós-excisão, devem ser revistas considerando novas pesquisas e sempre abordadas em função dos valores e expectativas das pacientes.

\section{Agradecimentos}

Agradecemos à nossa orientadora $\mathrm{Dr}^{\mathrm{a}}$ Denise Monteiro por toda a dedicação, carinho e aprendizado proporcionado. Obrigada!

\title{
A
}

\begin{abstract}
Cervical cancer is the second most common cancer among women worldwide, despite having great potential for prevention and cure when early diagnosed and treated, which can reduce the mortality rate among the affected. Since there is no consensus among the therapeutic measures in high grade cervical squamous intraepithelial lesions (HGSIL), we discuss its approach when dealing with adult women who have HGSIL and the follow-up after the adopted procedure. We performed electronic searches of MEDLINE (through PubMed), National Guidelines Clearinghouse, Google Scholar and Lilacs. The guidelines identified were evaluated according to their validity and recommendations. In relation to after-care, the European use cytology every 6 months, the Australians, cytology and colposcopy every 6 months, the Americans advocate the realization of hybrid capture in six to 12 months or cytology every 6 months. The Brazilian Guidelines Project, on its turn, recommends that clinical reassessments and Pap smear should be performed every three or four months during the first two years of follow-up. Studies comparing the method "See and Treat" with the three steps one (histology, colposcopy, biopsy) concluded that the latter is indicated for women ASCUS/LSIL before undergoing the excision of the transformation zone (ETZ), while the "View and Treat" is indicated in women with proven HGSIL in Cytology and suggestive in colposcopy, because it presents advantages such as low cost and immediate solution. All the guidelines are unanimous in stating that when facing proven HGSIL, excision of the injury through ablation or conization or ETZ is indicated.
\end{abstract}

Key words: Cervical intraepithelial neoplasia. Early detection of cancer. Therapeutics. Conization. Women.

\section{REFERENCIAS}

1. Brasil. Ministério da Saúde. Secretaria de Atenção à Saúde. Instituto Nacional de Câncer. Coordenação de Prevenção e Vigilância de Câncer. Estimativa 2010: Incidência de câncer no Brasil [online]. Rio de Janeiro: INCA, 2010. [acessado em 14 out. 2010]. Disponível em http://www.inca.gov.br/estimativa/2010/index.asp?link= tabelaestados.asp\&UF=BR

2. Koutsky L. Epidemiology of genital human papillomavirus infection. Am J Med 1997; 102(5A):3-8

3. Ministério da Saúde. Pesquisa sobre câncer de colo uterino [online]. Brasília: Ministério da Saúde, 2007. [Acessado em 12 mar. 2009]. Disponível em http://portal.saude.gov.br/portal/arquivos/pdf/ 2b_250311.pdf.

4. Brasil. Instituto Nacional de Câncer. Programa Nacional de Controle do Câncer do Colo do Útero e de Mama - Viva Mulher. Prevenção e detecção 2002 [online]. [Acessado em 09 abr. 2009]. Disponível em http://www.inca.gov.br/prevencao/programas/ pncccum/html.

5. Russomano F. Cirurgia de alta frequência [online]. Associação Brasileira de Genitoscopia. [Acessado em 25 out. 2009]. Disponível em http://www.colposcopy.org.br/espaco_mulher.php

6. Wright JD, Davila RM, Pinto KR, Merritt DF, Gibb RK, Rader JS, et al. Cervical dysplasia in adolescents. Obstet Gynecol 2005; 106(1):115-20.

7. Gardeil F, Barry-Walsh C, Prendiville W, Clinch J, Turner MJ. Persistent intraepithelial neoplasia after excision for cervical intraepithelial neoplasia grade III. Obstet Gynecol 1997; 89(3):419-22.

8. ACOG Pratice Bulletin No. 99: Management of abnormal cervical cytology and histology. American College of Obstetricians and Gymecologists. Obstet Gynecol 2008; 112(6):1419-44.
9. Sankasem A, Thavaramara T, Manusirivithaya S, Tangjitgamol S. Tumor persistence in high Ggrade squamous intraepithelial lesion patients with positive surgical margin post loop electrosurgical excision procedure. J Med Assoc Thai 2006; 89(7):934-40.

10. Jordan J, Arbyn M, Martin-Hirsch P, Schenck U, Baldauf JJ, Da Silva $D$, et al. European guidelines for quality assurance in cervical cancer screening: recommendations for clinical management of abnormal cervical cytology, part 1. Cytopathology 2008;19(6):34254.

11. Kyrgiou M, Koliopoulos G, Martin-Hirsch P, Arbyn M, Prendiville W, Paraskevaidis E. Obstetric outcomes after conservative treatment for intraepithelial or early invasive cervical lesions: systematic review and meta-analysis. Lancet 2006; 367(9509):48998.

12. Shin JW, Rho HS, Park CY. Factors influencing the choice between cold knife conization and loop electrosurgical excisional procedure for the treatment of cervical intraepithelial neoplasia. J Obstet Gynaecol Res 2009; 35(1):126-30.

13. Prendiville $\mathbf{W}$. The treatment of grade 3 cervical intraepithelial neoplasia. In: Prendiville W, Ritter J, Tatti S, Twiggs LB, editors. Colposcopy: management options. Edinburgh: Saunders; 2003. p.129-33

14. Wright TC Jr, Massad LS, Dunton CJ, Spitzer M, Wilkinson EJ, Solomon D; 2006 American Society for Colposcopy and Cervical Pathology-sponsored Consensus Conference. 2006 consensus guidelines for the management of women with cervical intraepithelial neoplasia or adenocarcinoma in situ. Am J Obstet Gynecol 2007; 197(4):340-5.

15. NHSCSP. Colposcopy and programme management: guidelines for the NHS Cervical Screening Programme. In: Luesley D, Leeson S, editors. NHS Cancer Screening Programmes, Sheffield: NHSCSP; 2004: p.1-80. 
16. Andrade JM, Yamaguchi NH, Oliveira AB, Perdicaris M, Pereira ST, Petitto JV, et al. Rastreamento, diagnóstico e tratamento do carcinoma do colo do útero. Projeto Diretrizes [online]; Maio de 2001. [Acessado em 20 mai. 2009]. Disponível em http://ncd.bvsalud.org/ lildbi/docsonline/2/5/052-Brazil-Cacu-Prof.pdf

17. Bodurka-Bevers D, Morris M, Eifel PJ, Levenback C, Bevers MW, Lucas KR, et al. Posttherapy surveillance of women with cervical cancer: an outcomes analysis. Gynecol Oncol 2000; 78(2):187-93.

18. American College of Obstetricians-Gynecologists. ACOG Committee Opinion. Evaluation and management of abnormal cervical cytology and histology in the adolescent, Number 330, April 2006. Obstet Gynecol 2006; 107(4):963-8.

19. Flannelly G, Bolger B, Fawzi H, De Lopes AB, Monaghan JM. Follow up after LLETZ: could schedules be modified according to risk of recurrence ? BJOG 2001; 108(10):1025-30.

20. Mitchell H, Hocking J. Influences on the risk of recurrent high grade cervical abnormality. Int J Gynecol Cancer 2002; 12(6):72834.

21. Jain S, Tseng CJ, Horng SG, Soong YK, Pao CC. Negative predictive value of human papillomavirus test following conization of the cervix uteri. Gynecol Oncol 2001; 82(1):177-80.

22. Lin CT, Tseng CJ, Lai CH, Hsueh S, Huang KG, Huang $H J$, et al. Value of human papillomavirus deoxyribonucleic acid testing after conization in the prediction of residual disease in the subsequent hysterectomy specimen. Am J Obstet Gynecol 2001; 184(5):9405.

23. Nobbenhuis MA, Helmerhorst TJ, van den Brule AJ, Rozendaal $L$, Voorhorst FJ, Bezemer PD, et al. Cytological regression and clearance of high-risk human papillomavirus in women with an abnormal cervical smear. Lancet 2001; 358(9295):1782-3.

24. Paraskevaidis E, Koliopoulos G, Alamanos Y, Malamou-Mitsi V, Lolis ED, Kitchener HC. Human papillomavirus testing and the outcome treatment for cervical intraepithelial neoplasia. Obstet Gynecol 2001; 98(5 Pt 1):833-6.

25. Nobbenhuis MA, Meijer CJ, van den Brule AJ, Rozendaal L, Voorhorst FJ, Risse EK, et al. Addition of high-risk HPV testing improves the current guidelines on follow-up after treatment for cervical intraepithelial neoplasia. Br J Cancer 2001; 84(6):796801.

26. Bar-Am A, Gamzu R, Levin I, Fainaru O, Niv J, Almog B. Follow-up combined cytology and human papillomavirus testing for patients post-cone biopsy: results of a long-term follow-up. Gynecol Oncol 2003:91(1):149-53.

27. Zielinski GD, Rozendaal L, Voorhorst FJ, Berkhof J, Snijders PJ, Risse EJ, et al. HPV testing can reduce the number of follow-up visits in women treated for cervical intraepithelial neoplasia grade 3. Gynecol Oncol 2003; 91(1):67-73.

28. Chao A, Lin CT, Hsueh S, Chou HH, Chang TC, Chen MY, et al. Usefulness of human papillomavirus testing in the follow-up of patients with high-grade cervical intraepithelial neoplasia after conization. Am J Obstet Gynecol 2004; 190(4):1046-51.

29. Cho H, Kim JH. Treatment of the patients with abnormal cervical cytology: a "see-and-treat" versus three-step strategy. J Gynecol Oncol 2009; 20(3):164-8.

30. Park JY, Bae J, Lim MC, Lim SY, Lee DO, Kang S, et al. Role of high risk-human papilloma virus test in the follow-up of patients who underwent conization of the cervix for cervical intraepithelial neoplasia. J Gynecol Oncol 2009; 20(2):86-90.

Recebido em 03/04/2010

Aceito para publicação em 05/05/2010

Conflito de interesse: nenhum

Fonte de financiamento: nenhuma

\section{Como citar este artigo:}

Oliveira PS, Coelho CC, Cerqueira EFS, Lopes FVF, Fernandes MSA, Monteiro DLM. Conduta na lesão intraepitelial de alto grau em muIheres adultas. Rev Col Bras Cir. [periódico na Internet] 2011; 38(4). Disponível em URL: http://www.scielo.br/rcbc

\section{Endereço para correspondência:}

Pauliana Sousa Oliveira

E-mail: paulianasousa@yahoo.com.br 